\title{
Fos-defined Activity in Rat Brainstem following Centripetal Acceleration
}

\author{
Galen D. Kaufman, ${ }^{1}$ John H. Anderson, ${ }^{2}$ and Alvin J. Beitz ${ }^{1}$ \\ 'Department of Veterinary Biology, University of Minnesota, St. Paul, Minnesota 55108 and ${ }^{2}$ Department of \\ Otolaryngology, University of Minnesota, Minneapolis, Minnesota 55455
}

To identify rat brainstem nuclei involved in the initial, shortterm response to a change in gravito-inertial force, adult Long-Evans rats were rotated in the horizontal plane for $\mathbf{9 0}$ min in complete darkness after they were eccentrically positioned off the axis of rotation (off-axis) causing a centripetal acceleration of $2 \mathrm{~g}$. Neural activation was defined by the brainstem distribution of the $\mathrm{c}$-fos primary response gene protein, Fos, using immunohistochemistry. The Fos labeling in off-axis animals was compared with that of control animals who were rotated on the axis of rotation (on-axis) with no centripetal acceleration, or who were restrained but not rotated. In the off-axis animals there was a significant labeling of neurons: in the inferior, medial, and y-group subnuclei of the vestibular complex; in subnuclei of the inferior olive, especially the dorsomedial cell column; in midbrain nuclei, including the interstitial nucleus of Cajal, nucleus of Darkschewitsch, Edinger-Westphal nucleus, and dorsolateral periaqueductal gray; in autonomic centers including the solitary nucleus, area postrema, and locus coeruleus; and in reticular nuclei including the lateral reticular nucleus and the lateral parabrachial nucleus. Also, there was greater Fos expression in the dorsomedial cell column, the principal inferior olive subnuclei, inferior vestibular nucleus, the dorsolateral central gray, and the locus coeruleus in animals who had their heads restrained compared to animals whose heads were not restrained. As one control, the vestibular neuroepithelium was destroyed by injecting sodium arsanilate into the middle ear, bilaterally. This resulted in a complete lack of Fos labeling in the vestibular nuclei and the inferior olive, and a significant reduction in labeling in other nuclei in the off-axis condition, indicating that these nuclei have a significant labyrinth-sensitive component to their Fos labeling. The data indicate that several novel brainstem regions, including the dorsomedial cell column of the inferior olive and the periaqueductal gray, as well as more traditional brainstem nuclei including vestibular and oculomotor related nuclei, respond to otolith activation during a sustained centripetal acceleration.

\footnotetext{
Received Mar. 2, 1992; revised June 5, 1992; accepted June 12, 1992.

We thank Jang-Hern Lee and Hung Nguyen for their supporting work in our labs. This work is made possible by a graduate student researcher program fellowship (NASA/NGT-50563) and NIH Grants DC01086, NS19208, DC00110, DE06682, and DA06687.

Correspondence should be addressed to Alvin J. Beitz, Department of Veterinary Biology, 295 AS/VM, University of Minnesota, 1988 Fitch Avenue, St. Paul, MN 55108.

Copyright (C) 1992 Society for Neuroscience $0270-6474 / 92 / 124489-12 \$ 05.00 / 0$
}

Normal coordination of body movement and posture depends upon an accurate spatial reference. The otolith organs of the vestibular labyrinth are sensitive to the direction and magnitude of the earth's gravity and could provide a reference for integrating vestibular, visual, and somatosensory information to help control movement. When a sustained change in the gravitoinertial force occurs (e.g., from a novel linear motion, in a microgravity environment, or as a result of inner ear pathology), a new reference for sensory integration must be established.

For the present experiments, we subjected rats to centripetal acceleration in order to create a change in the gravito-inertial force that stimulated the otolith organs of the inner ear. Fos immunohistochemistry was used to identify brainstem activity during this stimulus. There were two aims: (1) to establish whether Fos can be used as a neural activity marker in the vestibular system and to correlate these results with known anatomical and physiological data, and (2) to identify the brainstem nuclei that might take part in reestablishing an inertial reference. In contrast with earlier studies employing 2-deoxyglucose mapping studies of vestibular-induced activity (Sharp, 1976; Brizzee and Dunlap, 1983), the present investigation used a more precisely defined vestibular stimulus and a higher-resolution labeling method to identify single cells.

Our results indicate that Fos immunohistochemistry can be a useful tool for evaluating the short-term responses to vestibular stimulation and that head movement during rotation can affect the pattern of brainstem activation. We previously identified the strong activation of the dorsomedial cell column of the inferior olivary nucleus (DMCC; Kaufman et al., 1991) and here describe the expression sites of Fos throughout the brainstem of rats exposed to centripetal acceleration.

\section{Materials and Methods}

\section{Animal preparation}

All procedures were reviewed and approved by the University of Minnesota animal care committee, and adhered to guidelines for the use of animals in neuroscience research set forth by the Society for Neuroscience. Twenty-six male Long-Evans (pigmented) rats weighing approximately $200 \mathrm{gm}$ were used. Rats subjected to rotational stimuli were placed inside loosely fitting metal cones with their heads toward the apex of the cone. The cones were mounted on a scaffolding that could be lowered into a box mounted to the turntable. For the first trials (Kaufman et al., 1991), no head restraint was employed, but because there was head movement, all subsequent trials were performed with the head fixed by means of a screw embedded in a dental acrylic skull cap. The surgery for the skull cap was performed 4-7 d prior to the rotation session under ketamine/xylazine anesthesia $(95$ and $35 \mathrm{mg} / \mathrm{kg}$ i.m., respectively).

Several groups of animals served as controls. Control animals cxperienced no motion or only an angular acceleration that stimulated the 
horizontal semicircular canals but not the otolith organs of the inner ear. Room controls remained in their home cages in the same room as the centrifuge and for the same amount of time as those who were rotated. Surgery controls had the skull caps mounted 1 week prior to being killed. Surgery with restraint controls also had the skull cap but in addition were restrained inside a motionless cone (with no rotation) for $90 \mathrm{~min}$ in the dark and were then killed.

\section{Centripetal acceleration paradigm}

All rotation was in an earth horizontal plane at a constant angular velocity of $360 \% \mathrm{sec}$ and in a counterclockwise direction for $90 \mathrm{~min}$. Angular acceleration and deceleration were rapid $\left(\sim 50^{\circ} / \mathrm{sec} / \mathrm{sec}\right)$. Onaxis animals were situated $4 \mathrm{~cm}$ from the earth vertical axis of rotation, and off-axis animals were eccentric (46 or $54 \mathrm{~cm}$ ) from the axis of rotation (see Kaufman et al., 1991). The long axis of the body was tangential to the arc of rotation (perpendicular to the centripetal vector). Left or right orientation was defined as that side of the animal facing the center of rotation. The resultant of the gravitational and centripetal acceleration vectors had a magnitude of $1.02 \mathrm{~g}$ for the on-axis and 2.39 $g$ for the off-axis $(54 \mathrm{~cm})$ position. The direction of the resultant vector was $30^{\circ}$ down from the horizontal. It should be noted that the magnitude of the $g$ force was mild. Human centrifuge studies are often performed at a level of $3 \mathrm{~g}$ or greater, and rats can survive levels above $5 \mathrm{~g}$ for extended periods (Sudoh et al., 1987).

All rotations took place in a dark, light-tight room, during mid-morning, and $1-2 \mathrm{hr}$ after a typical $12 \mathrm{hr}$ light, $12 \mathrm{hr}$ dark cycle. Four rats were rotated at once: two on-axis and two off-axis. The animals were killed sequentially, beginning immediately after the $90 \mathrm{~min}$ rotation, so that the first rat was perfused within $10 \mathrm{~min}$ after the spin, and the last rat within $1 \mathrm{hr}$ afterward. A statistical analysis for a time effect on the amount of Fos labeling during the death period was not significant. After being rotated, the rats were left in the restraining cones and in the dark until they were perfused. Their behavior after the spin was calm. When they were removed from the cones, they resisted and showed no obvious signs of ataxia. The sequence of on-axis and off-axis animal perfusion was alternated.

\section{Fos immunohistochemistry}

Transcardial perfusion and postfixation. Immediately following each rotation, the animals were anesthetized one at a time with $\sim 1 \mathrm{ml}$ of chloral hydrate $(175 \mathrm{mg} / \mathrm{ml}$, i.p.) and, following a $5 \mathrm{~min}$ anesthetic induction, prerinsed transcardially with $100 \mathrm{ml}$ of $0.9 \%$ saline followed by $200-500 \mathrm{ml}$ of $4 \%$ paraformaldehyde in a phosphate-buffered saline solution (PBS; $0.05 \mathrm{M} \mathrm{Na}_{2} \mathrm{HPO}_{4}, 0.137 \mathrm{M} \mathrm{NaCl}, \mathrm{pH}$ 7.4). The entire brain was then removed, placed in $4 \%$ paraformaldehyde, and left there overnight at $4^{\circ} \mathrm{C}$. The following day the tissue was blocked and soaked in $30 \%$ sucrose/PBS for $2 \mathrm{~d}$ at $4^{\circ} \mathrm{C}$.

Immunohistochemical procedure. C-fos protein (Fos) antibody was obtained from Cambridge Biomedical (Wilmington, DE; catalog number OA-11-821, batch 02723). This sheep polyclonal was made against a 15 residue synthetic peptide (Met-Phe-Ser-Gly-Phe-Asn-Ala-Asp-TyrGlu-Ala-Ser-Ser-Ser-Arg-[Cys]) derived from a conserved region of human and mouse c-fos. Primary absorption, ELISA, and specificity testing for this antisera have been previously performed (Bullitt, 1989). No staining was observed on brainstem sections when the primary antibody was omitted from the protocol. It should be noted, however, that this antibody might also label Fos-related antigens (Dragunow and Faull, 1989). The sucrose-embedded brainstem tissue from each rat was sectioned at $30 \mu \mathrm{m}$ using a cryostat. Nonspecific binding sites were blocked with normal rabbit serum (1:50) for $30 \mathrm{~min}$ at room temperature (RT). Then, primary Fos antibody $(1: 8000)$ was applied overnight at $4^{\circ} \mathrm{C}$, followed by secondary antibody (rabbit anti-sheep IgG, 1:200) for $1 \mathrm{hr}$ at RT, and ABC (avidin-biotin complex, Vector, 1:100) for $1 \mathrm{hr}$ at RT. All antibodies and ABC were dissolved in PBS. Between each step, the tissue was rinsed three times with PBS ( $3 \mathrm{~min} /$ rinse) and the tissue was agitated on a rotator during each step. Next, endogenous peroxidase activity was destroyed by exposing the tissue to an ascending ethanol series $(50 \%, 70 \%, 50 \%$ ethanol for 10,15 , and $10 \mathrm{~min}$ at $\mathrm{RT}$ ), followed by rehydration in $0.05 \mathrm{M}$ Tris buffer for $10 \mathrm{~min}$. Finally, an intensified diaminobenzidine tetrahydrochloride (DAB) reaction (DAB, $0.02 \%$ $\mathrm{w} / \mathrm{v}$; nickel ammonium sulfate, $0.025 \% \mathrm{w} / \mathrm{v} ; \mathrm{H}_{2} \mathrm{O}_{2}, 0.002 \% \mathrm{v} / \mathrm{v}$; in Tris buffer, pH 7.4) was carried out for 20-30 min in the dark at RT. The sections were then mounted on gelatin-coated slides, dried, dehydrated, and coverslipped.

\section{Cell counting and statistical analysis}

The nomenclature and nuclear boundaries defined in the Paxinos and Watson (1986) stereotaxic rat brain atlas were utilized in this study. Transverse $30 \mu \mathrm{m}$ brainstem sections were evaluated with bright-field microscopy at $40 \times$ and $100 \times$ magnification. Only cells that had significant levels of DAB reaction product in their nucleus above that of tissue background levels were counted. Lightly immunolabeled cells were not counted. This was based on an average gray level cutoff of 130 units (out of 255) when the labeled neurons wcre analyzed with a digital image analysis system (UNIVERSAL IMAGING SOFTWARE). Contra- and ipsilateral nuclei were counted individually in each animal, and the two highest counts per nucleus for each side at a defined bregma location were recorded, giving four values per nucleus for a given condition. For an analysis of possible asymmetries, the values for the left nucleus of rats in the left orientation were pooled with the values for the right nucleus of rats in the right orientation, and these values were compared to those of the opposite nuclei using a one-way analysis of variance (ANOVA). In all other analysis, the data from both sides for a given experimental condition were pooled together and a one-way ANOVA (STATVIEW 512+ software, Brainpower Inc., Abacus Concepts Inc., 1986) was performed using those four values for each rat. The Scheffe $F$ test was used to determine probability values. Only two conditions were evaluated at a time (e.g., on-axis vs. off-axis). Note that for clarity of presentation in the figures, the standard error (based on an average value for each rat for a given experimental condition), and not the standard deviation of the four values for each rat, is shown (see Figs. 1, 2,6).

\section{Labyrinth ablation controls}

Fos immunolabeling in the off-axis animals could be a result of an increase in proprioceptive inputs and/or cutaneous stimulation due to the centripetal acceleration. To demonstrate which components of the Fos labeling were actually labyrinth in origin, we attempted to destroy the vestibular neuroepithelium in several rats. Initially we tried using the ototoxic aminoglycoside gentamicin sulfate $(10 \mathrm{or} 25 \mathrm{mg} / \mathrm{kg}$, i.p. once a day for $21 \mathrm{~d}$ ), but there were no behavioral signs of vestibular dysfunction, consistent with previous reports showing no histological damage unless a very high dose, $100 \mathrm{mg} / \mathrm{kg} / \mathrm{d}$ for $30 \mathrm{~d}$, was used (cf. Tran Ba Huy et al., 1986). Thereafter, we injected sodium arsanilate $(0.05 \mathrm{ml}$ of a $100 \mathrm{mg} / \mathrm{ml}$ arsanilic acid solution in normal saline and titrated to $\mathrm{pH} 7.6)$ through the tympanic membrane into the middle ear bilaterally (total dose was $50 \mathrm{mg} / \mathrm{kg}$ ) of three rats to destroy the vestibular neuroepithelium. Four days following the injections, these rats were subjected to the centripetal stimulus. The efficacy and rapidity of this method have been outlined (Horn et al., 1981); at $50 \mathrm{mg} / \mathrm{kg}$, the procedure creates a labyrinthectomy that seems to be permanent in Long-Evans rats. The oral $\mathrm{LD}_{50}$ for sodium arsanilate in male rats is $>1000 \mathrm{mg} / \mathrm{kg}$.

Histology was obtained for some of the labyrinthectomy controls. Following perfusion, the temporal bones were removed and placed in a decalcification solution (Decalcifier I, Surgipath Medical Ind., Inc.) for I week. The tissue was then rinsed through a graded alcohol series, xylene, and embedded in paraffin for sectioning. Sections $7 \mu \mathrm{m}$ thick were mounted on subbed slides, heated briefly, dried overnight, and stained with hematoxylin and eosin.

\section{Results}

A description of the Fos labeling will be presented for different groups of animals and stimulus conditions in the following sections: (1) no rotation and on-axis (normal control animals), (2) off-axis (normal animals with the head restrained), (3) labyrinthectomy (arsanilate-injected control animals), and (4) head free (off-axis animals whose heads were not restrained during the rotation). Within each section, the description of Fos immunolabeling will be given for the following three groups of brainstem nuclei whose labeling depended on the experimental condition: (1) vestibular nuclei: the medial, inferior, $\mathrm{x}$ - and y-group vestibular nuclei; (2) vestibular projection and precerebcllar $n u^{-}$ clei: the inferior olive subnuclei $\beta$, principal cell column, and DMCC, the prepositus hypoglossi; spinal trigeminal nucleus; interstitial nucleus of Cajal; and the Darkschewitsch nucleus; 

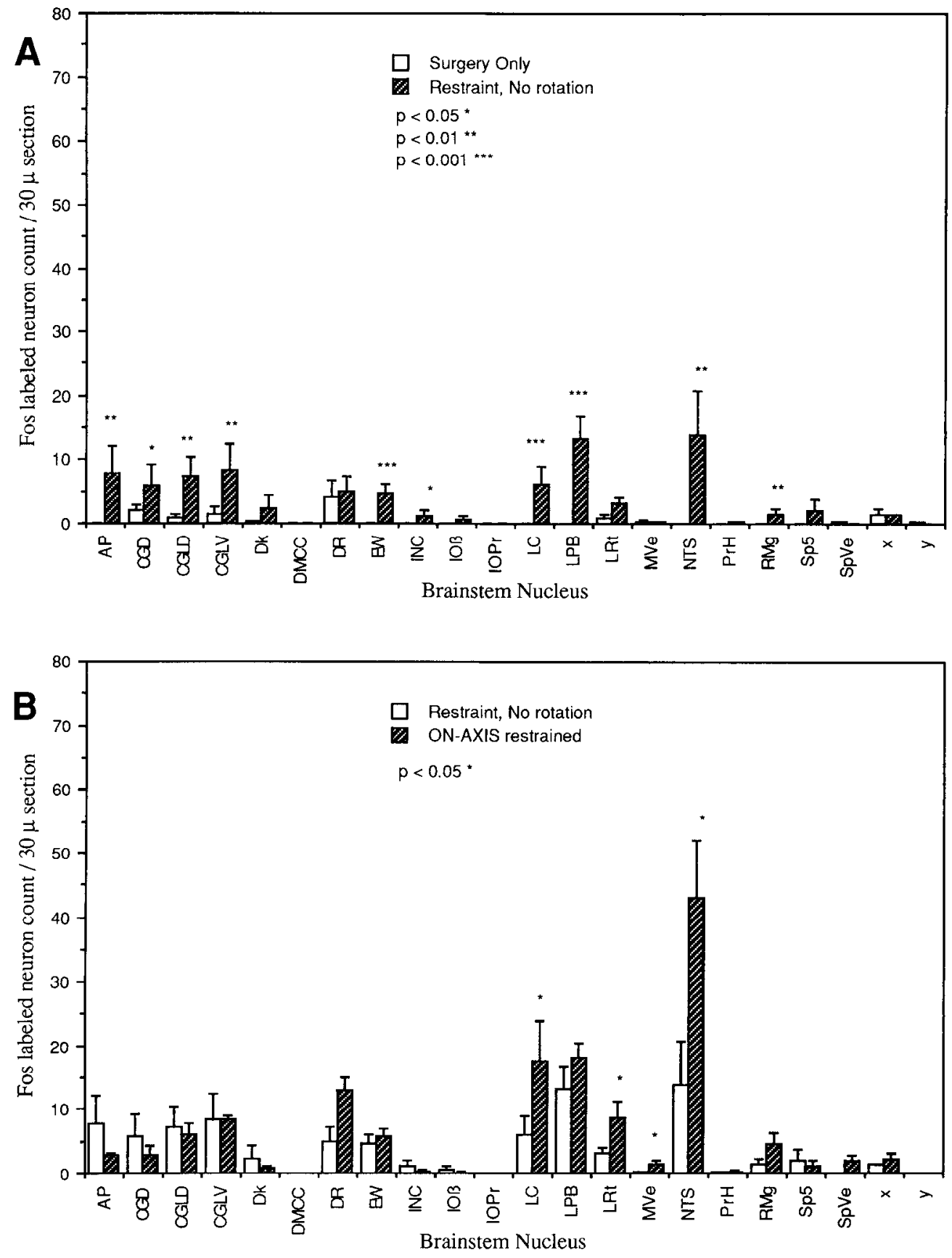

Figure 1. A, Fos-labeled neuron counts in brainstem nuclei of control rats that had skull cap surgery only, and those that had the surgery and 90 min of head restraint in the dark. $B, A$ comparison of Fos-labeled neuron counts between rats whose heads were restrained in the dark but without any rotation and rats rotated on-axis. $p$ values are based on the Scheffé $F$ test. Note that an absent column for some brainstem nuclei means that there was no Fos labeling for that condition. $A P$, area postrema; $C G D, C G L D$, and $C G L V$, dorsal, dorsolateral, and lateroventral periaqueductal gray, respectively; $D k$, nucleus of Darkschevich; $D M C^{\prime} C$, dorsomedial cell column of the inferior olive; $D R$, dorsal raphe; $E W$, Edinger-Westphal nucleus; $I N C$, interstitial nucleus of Cajal; $I O \beta$, inferior olivary beta subnucleus; $I O P r$, inferior olivary principal subnucleus; $L C$, locus coeruleus; $L P B$, lateral parabrachial nucleus; $L R t$, lateral reticular nucleus; $M V e$, medial vestibular nucleus; $N T S$, solitary nucleus; $\operatorname{PrH}$, prepositus hypoglossi; $R M g$, raphe magnus; $S p 5$, spinal trigeminal nucleus; $S p V e$, inferior (spinal) vestibular nucleus; $x$, x-subgroup of vestibular nuclei; $y, y$-subgroup of vestibular nuclei. and (3) raphe, reticular, and autonomic nuclei. The raphe magnus and the dorsal raphe; the dorsolateral and ventrolateral periaqueductal gray, the lateral parabrachial nucleus, and the lateral reticular nucleus; and the area postrema, solitary nucleus, locus coeruleus, and Edinger-Westphal nucleus.

In addition to these areas, there were several brainstem nuclei that exhibited Fos immunolabeling regardless of the experimental condition. These included the cochlear nuclei, superior olivary nuclei, and the inferior colliculus (consistent with previous 2-deoxyglucose work; Schwartz and Sharp, 1978); the supramammillary, arcuate, trapezoid body, and reticulotegmental nuclei; and the ventral and alpha areas of the periaqueductal gray. In several animals, we observed Fos immunolabeling in discrete areas of both the molecular and granular layers of the cerebellum, consistent with a 2-deoxyglucose study using vestibular stimulation (Sharp, 1976), but details of this are beyond the scope of this article.

\section{No rotation}

In this group of animals, the aim was to establish baseline levels of Fos labeling attributable to constitutive expression and/or the stress of restraint, independent of centripetal acceleration. Both the room control $(n=3)$ and surgery control $(n=3$; no restraint) animals exhibited little Fos immunolabeling, and that which was observed occurred in nuclei known from previous experience and other studies to display constitutive Fos expression (Bullitt, 1990). This included the central gray, reticular, and raphe areas, and pontine nuclei where a few to moderate 


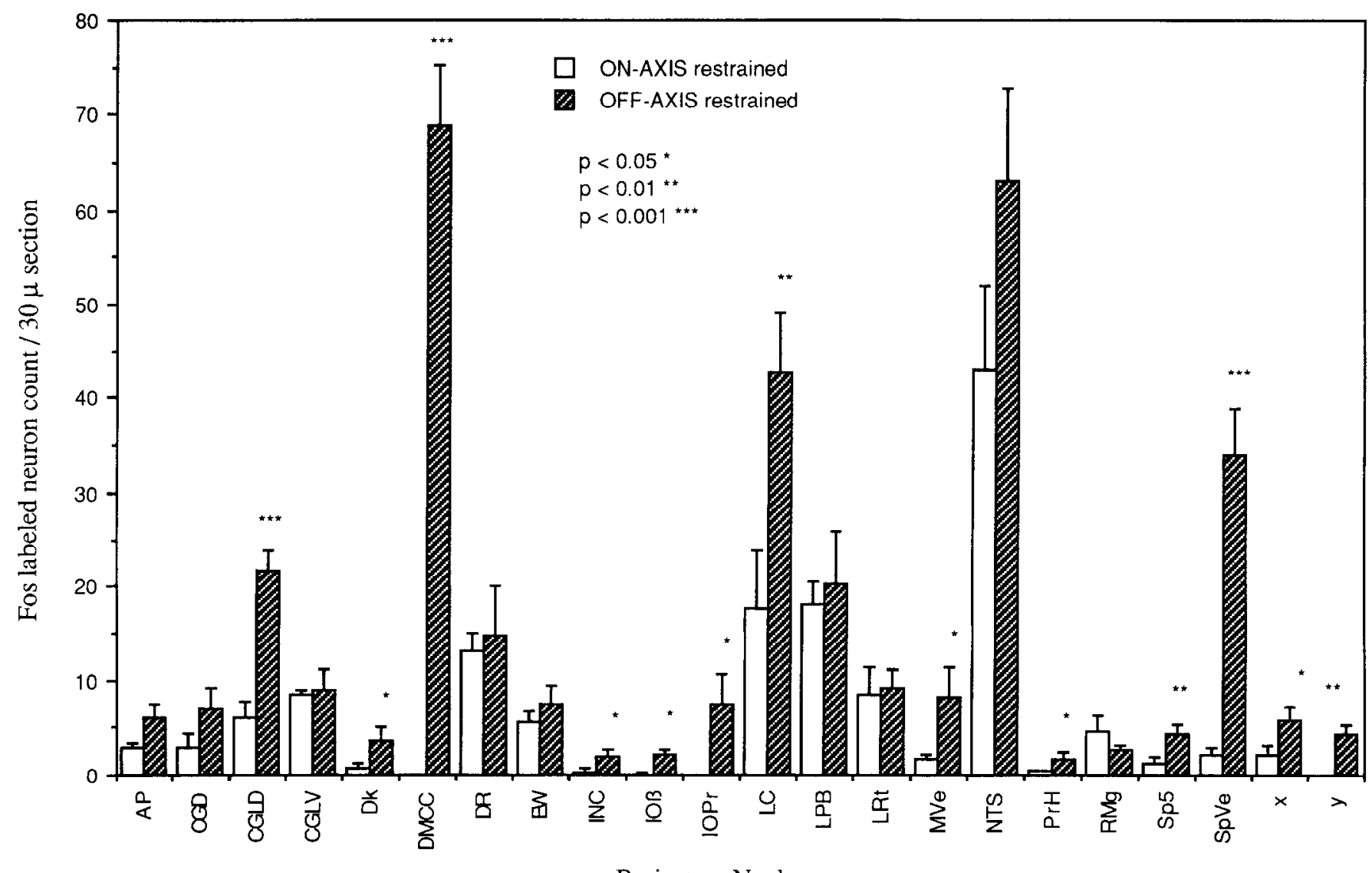

Figure 2. Fos labeling in brainstem nuclei in rats positioned on-axis and off-axis. All rats had their heads restrained during the rotation. Quantification is based on the absolute number of cells that were labeled. Asterisks refer to the probability levels for significant differences between off-axis and on-axis conditions. Abbreviations are as in Figure 1.

numbers of immunolabeled neurons were observed. In addition, occasional immunolabeled neurons were evident in other areas, but no consistent patterns emerged. The surgery with restraint group ( $n=3$ ) exhibited increases in Fos immunolabeling in many nuclei. Figure $1 A$ compares Fos-labeled neuron counts in several nuclei for the surgery only and surgery with restraint groups. The following was observed in the restrained animals.

Vestibular nuclei. No significant labeling was observed in the vestibular nuclei, consistent with a presumed lack of vestibular stimulation.

Vestibular projection and precerebellar nuclei. A few labeled neurons were seen in the interstitial nucleus of Cajal and Darkschewitsch nucleus.

Raphe, reticular, and autonomic nuclei. Labeling of the dorsal raphe, raphe magnus, lateral parabrachial nucleus, and all areas of the periaqueductal gray, was evident. The locus coeruleus, Edinger-Westphal nucleus, area postrema, and solitary nucleus all showed moderate labeling, consistent with a general activation of the autonomic nervous system.

\section{On-axis}

On-axis animals $(n=4)$ served as an important control for animals that were subjected to centripetal acceleration in the off-axis position. The rats experienced the brief angular accelerations of 7-8 sec when the centrifuge increased its angular velocity from zero to $360 \% \mathrm{sec}$ and when it decelerated back to zero. With the head restrained, the neural activity related to this acceleration should last on the order of only 1-2 min. The prolongation beyond the duration of the acceleration is due to the brainstem integration of the vestibular afferent activity (Robinson, 1981). In on-axis animals, several nuclei had an increase in Fos expression when compared to the nuclei of rats that were restrained but were not rotated. This might indicate that the brief angular acceleration or the small amount of centripetal acceleration $(\sim 0.15 \mathrm{~g})$ activated some neurons sufficiently to express Fos. Figure $1 B$ shows the differences in Fos neuron counts for those rotating on-axis compared to rats restrained without rotation. The following can be noted.

Vestibular nuclei. The medial and inferior vestibular nuclei showed only a slightly greater number $(p<0.08)$ of Fos-labeled neurons.

Vestibular projection and precerebellar nuclei. No significant differences were noted.

Raphe, reticular, and autonomic nuclei. The lateral reticular nucleus had a significant increase $(p<0.05)$ and the dorsal raphe had a slight increase $(p<0.06)$ in Fos labeling. The solitary nucleus and locus coeruleus exhibited significant increases $(p<0.05)$ in Fos.

\section{Off-axis}

A comparison between on-axis $(n=4)$ and off-axis $(n=5)$ animals was made in order to quantify the effect of hypergravity stimulation. Figure 2 shows a graph of Fos neuron counts for both on-axis and off-axis rats. For an overview, Figure 3 shows 

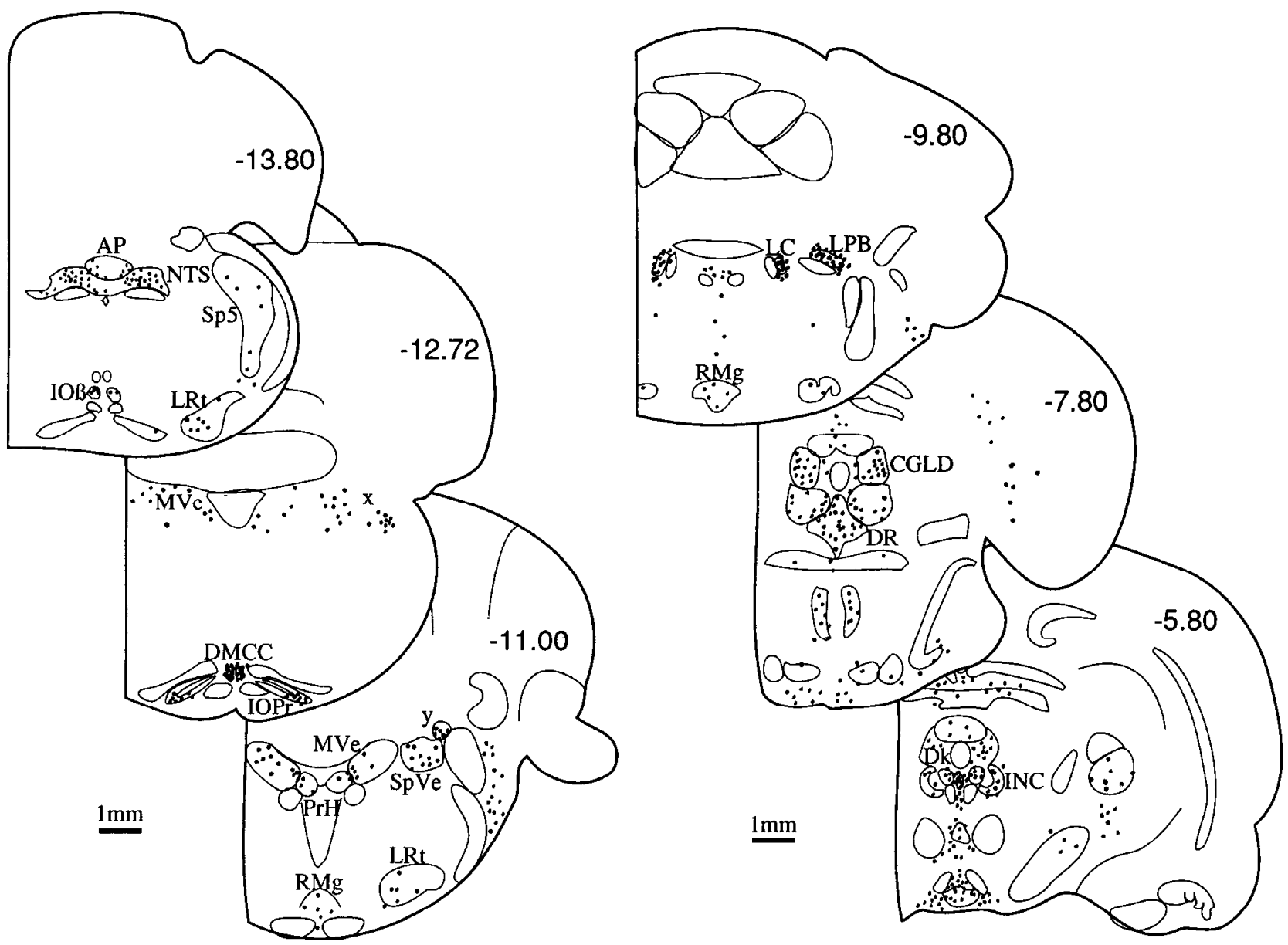

Figure 3. Camera lucida drawings of coronal sections showing the Fos labeling in one rat in the off-axis position. The numbers refer to the level of the section with respect to Bregma in millimeters. Abbreviations are as in Figure 1.

a camera lucida drawing of approximate Fos labeling in one offaxis rat. The following nuclei should be noted.

Vestibular nuclei. The pattern of activation was equable with anatomical studies delineating the projection of otolith afferents, provided the caveats associated with Fos expression are taken into account (Dragunow and Faull, 1989). In this regard, only occasional labeled cells were seen in the lateral and superior vestibular nuclei, although previous tracing studies (cf. ButtnerEnncver, 1988) have shown that the lateral nucleus receives a direct otolith afferent projection. Also, we observed labeling farther caudally in the vestibular complex than direct projections of otolith afferents. There was a consistent, significant labeling of the medial vestibular nucleus, inferior vestibular nucleus, and y-group (Fig. $4 B$ ), nuclei that receive a strong otolith input (cf. Buttner-Ennever, 1988). There was also labeling of a small group of cells at the border between the medial vestibular nucleus and the prepositus hypoglossi near the fourth ventricle (Fig. 4A). The F group of the vestibular complex (Mehler and Rubertone, 1985) was not well delineated from the inferior vestibular nucleus in unstained sections, and immunolabeled cells in this area were included with the inferior vestibular nucleus. The x-group, a column of cells bordering the entire inferior vestibular nucleus laterally, seemed to label most strongly in its caudal part in off-axis animals.

Vestibular projection and precerebellar nuclei. As reported previously (Kaufman et al., 1991), one of the most striking Fos- labeled areas in off-axis animals was the DMCC of the inferior olivary nucleus, a subnucleus that receives heavy vestibular and vestibular-related nuclei projections (Anderson et al., 1983; Swenson and Castro, 1983). In animals whose heads were restrained during the rotation, statistically significant Fos labeling was also observed in two other inferior olivary subnuclei: the $\beta$-subnucleus and the caudal lateroventral principal subnucleus, areas that receive projections from, among others, visual and cerebral cortical structures, respectivcly (Azizi and Woodward, 1987). These latter subnuclei were unlabeled in animals in the on-axis condition, and even in the off-axis condition the number of labeled neurons was small, and there was variability from animal to animal, in marked contrast with the strong DMCC immunolabeling in all off-axis animals. The DMCC labeling was strong both in absolute numbers and as a percentage of total neurons in the nucleus.

Other brainstem nuclei that were significantly labeled in the off-axis animals were the spinal trigeminal nucleus; the interstitial nucleus of Cajal, a perioculomotor nucleus known to help control vertical and torsional eye movements and to receive an otolith input (Fukushima, 1987; McCrea et al., 1987; Ostrowska et al., 1990; Crawford et al., 1991); and the Darkschewitsch nucleus.

Raphe, reticular, and autonomic nuclei. Among this group, the majority of labeled neurons occurred in the pcriaqucductal gray. When this area was analyzed according to the four major 

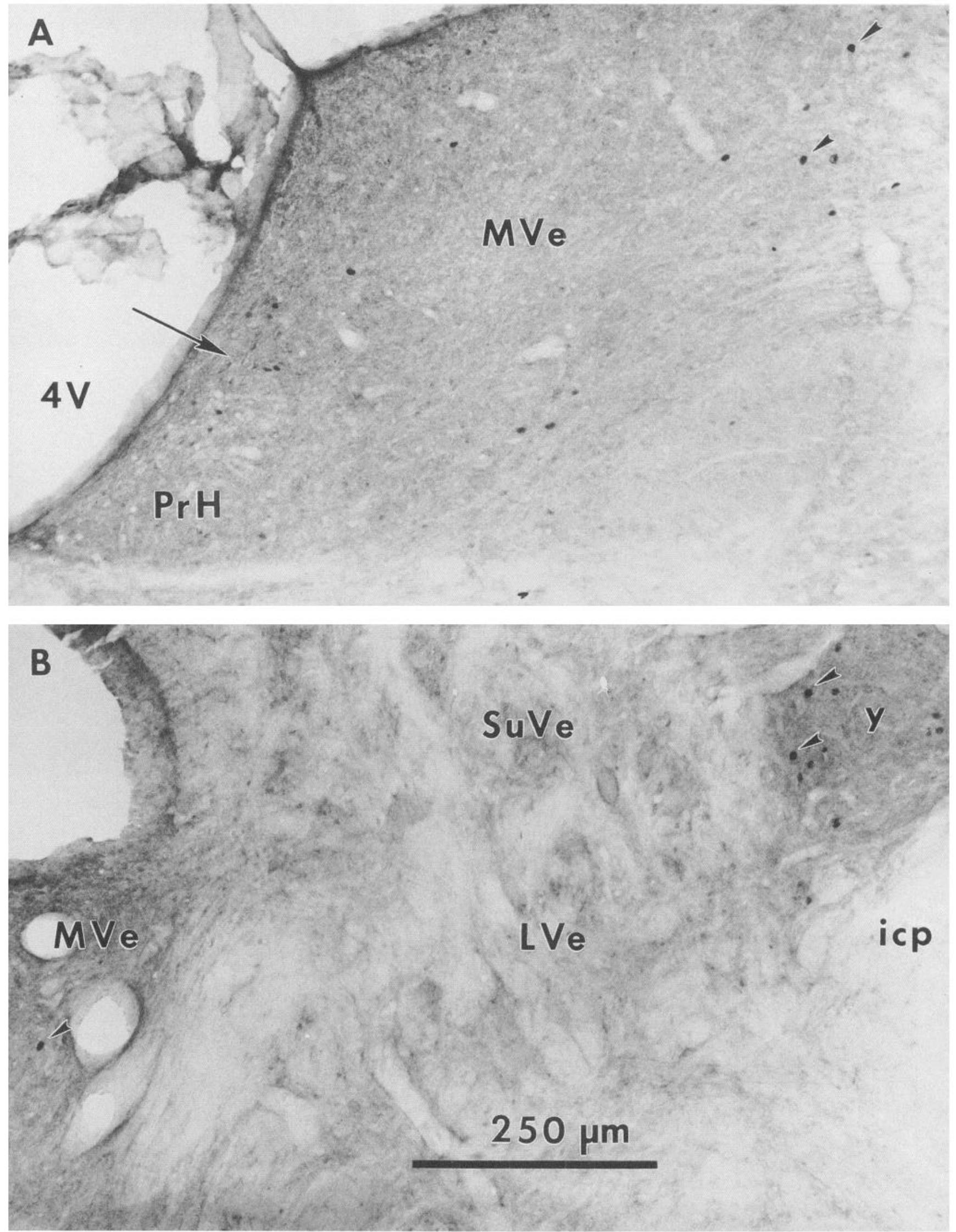

Figure 4. Photomicrograph of Fos immunolabeling in the vestibular complex of one rat subjected to off-axis rotation. $A$, Prepositus hypoglossi $(\mathrm{PrH})$ and medial vestibular nucleus $(\mathrm{MVe})$ show Fos labeled neurons. The arrowheads denote labeled neurons in the $M V e$. The arrow points to a group of neurons that were consistently labeled in off-axis animals and were situated on the border between $\operatorname{PrH}$ and $M V e$. B. Note the lack of labeling in the lateral $(L V e)$ and superior $(S u V e)$ vestibular nuclei, but the presence of labeling in the y-group $(y)$ and $M V e(a r r o w h e a d s)$; icp, inferior cerehellar neduncle 


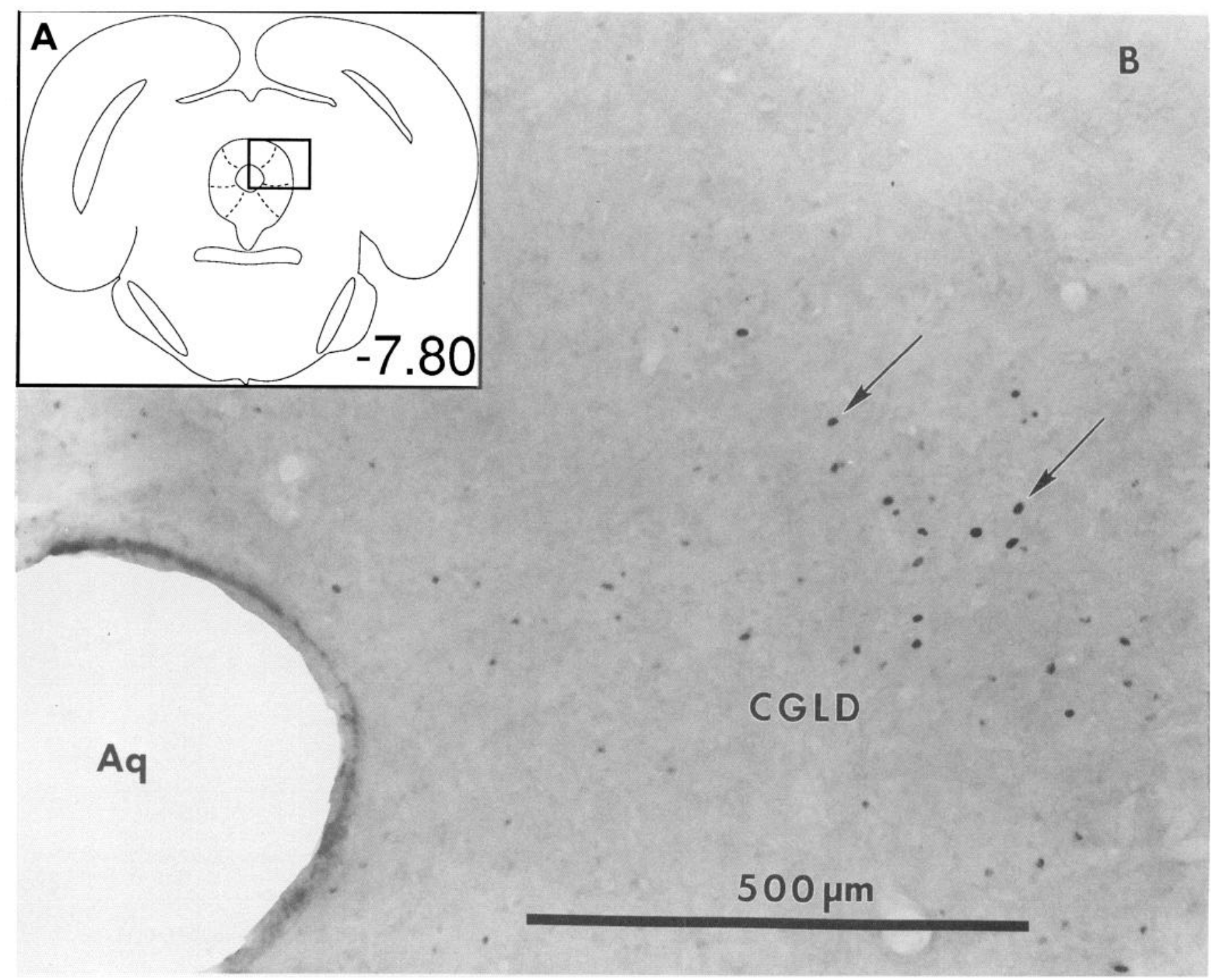

Figure 5. A, Inset showing area of micrograph in the midbrain. B. Fos immunolabeling in the dorsolateral periaqueductal gray $(C G L D)$ in an offaxis rat. The head was restrained during the rotation. Arrows show strongly labeled cells. $A q$, mesencephalic aqueduct.

subdivisions (i.e., medial, dorsal, dorsolateral, and lateroventral subnuclei; Beitz, 1985), we found a significant activation only in the dorsolateral subdivision (Fig. 5). Both the area postrema and solitary nucleus showed a slight, but nonsignificant, increase in labeling in the off-axis animals compared to the on-axis controls (Fig. 2). In the area postrema, most labeling was at the junction between the area postrema and the solitary nucleus, an area referred to as the area subpostrema in other species (D’Amelio et al., 1987).

\section{Labyrinthectomy}

Within $24 \mathrm{hr}$ after injecting sodium arsanilate into the middle ear bilaterally, rats displayed severe ataxia, head tremor, a widebased stance, and a tendency to back up vigorously when first placed on a hard surface. This behavior persisted until the rats were killed 3-4 d later. However, after $4 \mathrm{~d}$ there was evidence of compensation, characterized by better coordination, grooming, and the ability to hold the head still for short periods of time. At $4 \mathrm{~d}$ postinjection, these rats were subjected to the rotation, resulting in much less Fos immunolabeling compared to normal rats in many nuclei. Figure 6 shows the Fos counts for the normal and arsanilate animals after off-axis rotation. Labeling in the DMCC and the vestibular nuclei was completely abolished in the arsanilate-treated rats. In addition, only an occasional neuron was labeled in the area postrema, the solitary nucleus had an approximately $80 \%$ reduction in labeling, and there were significant reductions in the dorsolateral periaqueductal gray, interstitial nucleus of Cajal, Darkschewitsch nucleus, and the locus coeruleus.

Temporal bone histology from the arsanilate rats revealed neuroepithelial cell pathology throughout the cochlear, semicircular canal, and otolith organ tissue. This was characterized by degeneration of the supporting stroma, vacuolation of the epithelial cells, collapse and thickening of the epithelial layer, infiltration by inflammatory cells, and exudation within the cochlear and canal lumens. Figure 7 shows a high-magnification $(1000 \times)$ bright-field micrograph of otolith organ hair cells from a normal rat and an arsanilate-treated rat.

There was no vestibular dysfunction, behaviorally, in one rat injected with saline only into the middle ear, bilaterally. After that animal was subjected to the off-axis rotation, the resulting labeling was identical to that of the normal animals. An iden- 


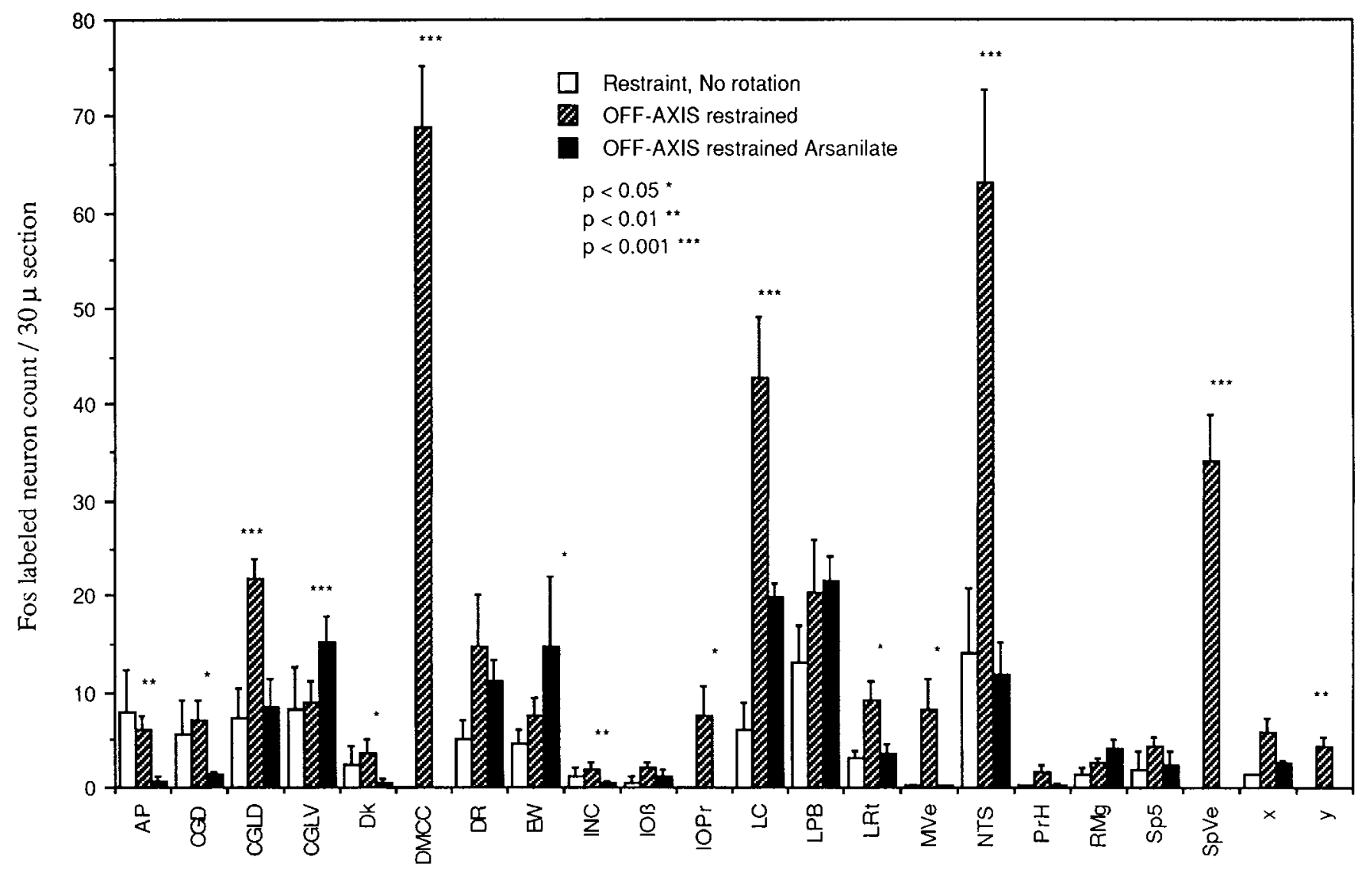

Brainstem Nucleus

Figure 6. The differences in Fos labeling between normal off-axis rats with their heads restrained and those who had arsanilate injections. Also shown is the amount of labeling in rats that were only restrained in the dark but were not rotated. In several nuclei, the arsanilate labyrinthectomy significantly reduced the labeling. $p$ values are based on the ANOVA comparing normal and arsanilate off-axis rats only. Abbreviations are as in Figure 1.

tical, but subcutaneous dose of arsanilate given to another rat failcd to clicit any bchavioral symptoms, and the animal's Fos labeling was identical to normal room controls. Another rat that had the arsanilate injected into the middle ear bilaterally was subjected to the on-axis rotation. The resulting labeling was reduced in most areas compared to normal on-axis animals.

\section{Head free}

There were several significant differences between the off-axis animals with the head free to move $(n=4)$ and the off-axis animals with the head fixed to the restraining cone $(n=5)$.

Vestibular nuclei. The inferior vestibular nucleus had a significant decrease in Fos immunolabeling in animals who had their heads free during the rotation.

Vestibular projection and precerebellar nuclei. The DMCC had a modest but statistically significant decrease in the head-free animals compared to the restrained animals. In previous experiments (Kaufman et al., 1991) the animals' heads were not restrained, and it was found that no other inferior olivary subnucleus was immunolabeled. However, in animals whose heads were restrained, there was some labeling in other subnuclei: the $\beta$ - and principal subnucleus, and to a lesser extent in the B and $C$ subnuclei.

Raphe, reticular, and autonomic nuclei. In animals with the head free, the dorsolateral periaqueductal gray had statistically less labeling, and the lateroventral periaqueductal gray had sig- nificantly more labeling. Also, the locus coeruleus had very significantly less $(p<0.001)$ labcling, and there was a slight, but not significant increase in the area postrema, and a slight but not significant decrease in the solitary nucleus.

\section{Discussion}

The present results show that the DMCC, the y-group and inferior vestibular nuclei, and the dorsolateral periaqueductal gray were strongly labeled for Fos $(p<0.01)$ following a hypergravity stimulus. The medial vestibular nucleus, $x$-group, and other areas of the inferior olive also were significantly labeled ( $p<$ 0.05 ). Increases in the area postrema and solitary nucleus and several raphe and reticular nuclei indicate that those areas might be involved as a result of the stress of being restrained. Following a discussion of the arsanilate labyrinthectomy, and the head restraint controls, the possible functional significance of these findings will be addressed.

\section{Arsanilate labyrinthectomy}

In the off-axis condition, rats that had sodium arsanilate injected into the middle ear bilaterally had a decrease or lack of Fos labeling in many nuclei that were labeled in normal rats (see Fig. 6). The level of Fos immunolabeling was similar to that observed in normal rats that were restrained without being rotated. This is strong evidence that those areas were activated by the vestibular stimulation in the normal rats, and that the 

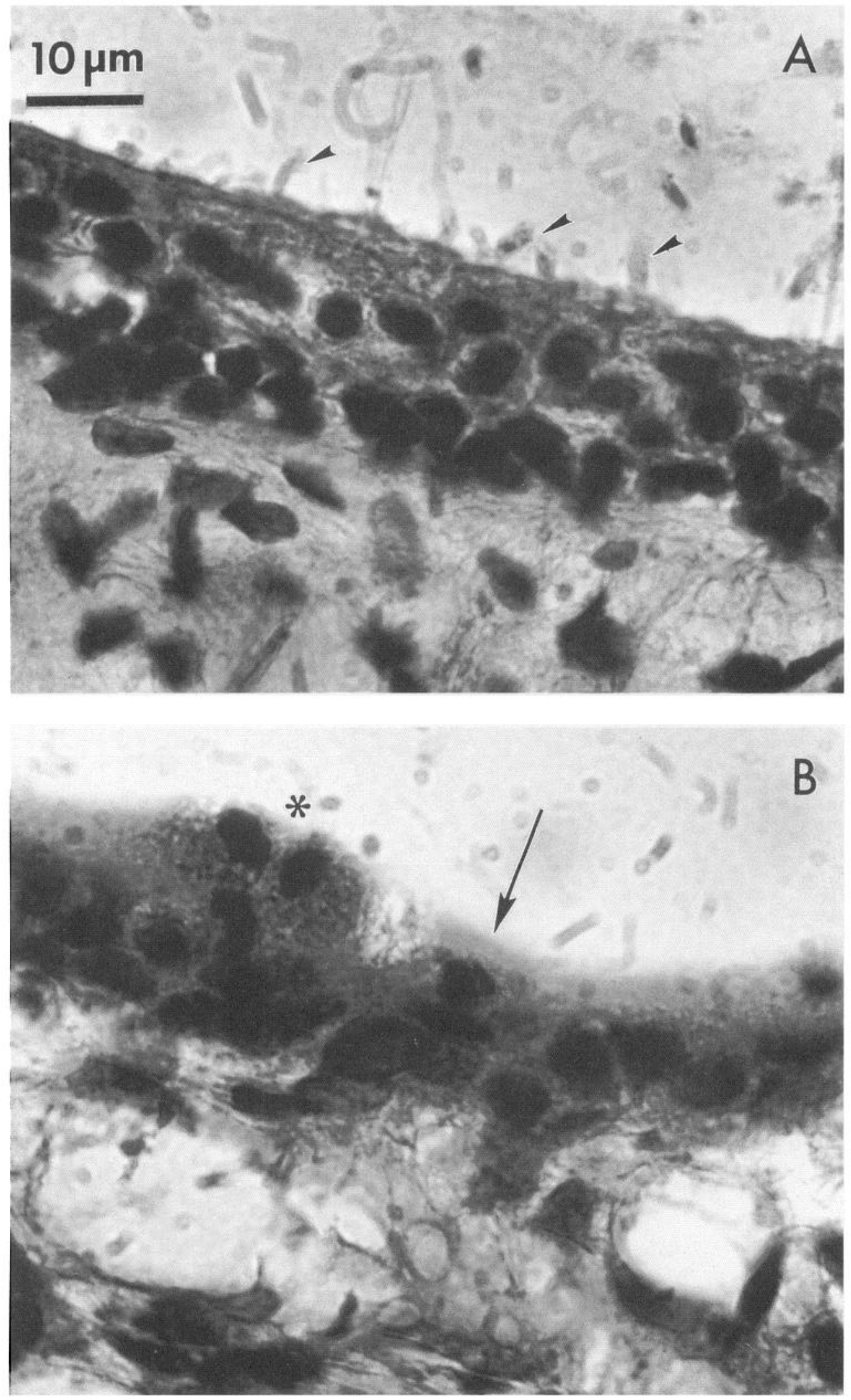

Figure 7. Photomicrograph $(1000 \times)$ of otolith organ neuroepithelium from a normal rat $(A)$, and a rat that had sodium arsanilate injected into the middle ear cavity $(B)$. The arrowheads in $A$ show a few cilia that survived the histology procedures. The asterisk in $B$ is adjacent to two inflammatory-type cells, probably macrophages, that were present throughout the damaged epithelium. The arrow points to a layer of exudate over the collapsed columnar epithelium.
Fos expression was superimposed upon a level of expression that was due to other factors in the experimental setup. One rat was given arsanilate and then subjected to the on-axis protocol. There was a reduction in labeling compared to normal rats in the on-axis condition, indicating that some of the Fos labeling in the on-axis condition might also be a result of labyrinth stimulation (i.e., due to the angular acceleration/deceleration at the beginning/end of rotation or to the small amount of centripetal acceleration, $\sim 0.15 \mathrm{~g}$ ).

The failure to elicit any signs of vestibular dysfunction after 
an identical subcutaneous dose of arsanilate indicates that there were no systemic effects and is consistent with previous work by Horn et al. (1981), who used much greater doses over many days before seeing behavioral evidence of vestibular dysfunction in Long-Evans rats. Histology of the vestibular labyrinth in the arsanilate-injected rats showed that portions of the vestibular nerve and spinal ganglia did not have pathological changes at the level of light microscopy. This suggests that the effect of the arsanilate was on the inner ear epithelia only (see Cass et al., 1989; Cass and Goshgarian, 1991).

\section{Stress and head restraint}

The finding that most reticular and raphe nuclei were significantly immunolabeled in both the on-axis and off-axis animals suggests that environmental factors other than the vestibular (i.e., otolith) stimulation were important for these nuclei. A wide body of evidence indicates that the dorsal raphe, raphe magnus, and lateroventral part of the periaqueductal gray are involved with the modulation of pain (Beitz, 1991), and the fact that there are strong correlations between pain and certain kinds of stress suggests that these areas were not responding directly to the vestibular stimulation. In particular, it should be noted that the lateroventral periaqueductal gray, an area known to be involved with pain modulation, cardiovascular control, and defense responses (Zhang et al., 1990; Beitz, 1991), showed an increase in Fos labeling in off-axis animals with heads free compared with those whose heads were restrained. This might be due to stress-related responses caused by the complex vestibular stimulus resulting from head movement while rotating, and not a direct vestibular response. The fact that the lateroventral periaqueductal gray showed an increase in labeling after the arsanilate injection in off-axis animals is consistent with this possibility.

Head movements while an animal is rotating would stimulate the semicircular canals by bringing a given canal into or out of the plane of rotation and change the direction of the shear forces acting on the otolith organs. As a result, there would be an interaction of otolith and semicircular canal dependent activity in the brainstem. Corcoran et al. (1989) suggested that rhesus monkeys were more resistant to motion sickness than squirrel monkeys because the rhesus monkeys quickly learned to keep their head still when in the off-axis situation, while squirrel monkeys did not. This behavioral strategy could be used to minimize vestibular stimulation and might correlate with the effect of head restraint in the present experiments. Most nuclei revealed greater Fos expression in rats with the head restrained. The constant stimulus associated with a restrained head $(90 \mathrm{~min}$ of centripetal but no angular acceleration) could act to increase Fos expression and create a brainstem activity map with sharper definition. In addition, it is possible that attempted head movements with the restraint might be a contributing factor.

\section{Functional significance of specific brainstem nuclei}

Dorsolateral periaqueductal gray. The off-axis activation of the dorsolateral periaqueductal gray supports the recent anatomical work by Holstege and Cowie (1990), which suggests that the dorsolateral periaqueductal gray might receive spatial orientation inputs from the prepositus hypoglossi, lateral substantia nigra pars reticulata, and the intermediate and deep layers of the superior colliculus. In contrast, this subdivision of the periaqueductal gray does not receive inputs from the basal (limbic) forebrain, which heavily projects to the remainder of the periaq- ueductal gray. The dorsolateral gray is known to respond to saccade activity (Kase et al., 1986) and receive fastigial nucleus input (Hirai et al., 1982). Previous studies (Beitz, 1982; Meller and Dennis, 1986) found that the hypothalamus and cuneate nuclei also project strongly to the dorsolateral area. Holstege and Cowie (1990) proposed that the dorsolateral periaqueductal gray influences the other periaqueductal subnuclei and the limbic system to help control appropriate behavioral responses to novel environmental conditions, which might involve integrating vestibular and visual inputs.

Area postrema and solitary nucleus. Area postrema has been implicated as a trigger zone for motion-induced emesis in the dog (Wang and Chinn, 1954) and monkcy (Brizzce et al., 1980). However, more recent results are not as definite regarding its functional role (Borison and Borison, 1986; Wilpizeski et al., 1986; Sutton et al., 1988; Fox et al., 1990), and precisely how the area postrema affects vestibular reflex responses is still controversial (Jovanovic-Micic and Strbac, 1989; Gallo et al., 1991). Interaction of the area postrema with other areas, for example, the solilary nucleus (Hay and Bishop, 1991), might be involved with homeostatic mechani ns controlling cardiovascular responses and electrolyte balance. In our experimental condition, the effects of motion on systemic circulation and vagal activity might have affected the solitary nucleus, and the labeling increase in the area postrema could be a result of that. Another possibility is an increase in the activity of other areas known to project to the area postrema, for example, the hypothalamus (Larsen et al., 1991). However, our data also suggest that vestibular stimulation might be involved. Off-axis animals with their heads free had slightly higher labeling in the area postrema than those with the head fixed. Since any head movement during rotation would have resulted in transient stimulation of different semicircular canals, and this can cause motion sickness in primates, the data suggest that the area postrema might have responded to the complex vestibular stimulus. The finding that off-axis arsanilate-labyrinthectomized rats showed greatly reduced Fos labeling in both the area postrema and the solitary nucleus is consistent with this possibility.

Vestibular nuclei. The pattern of labeling in the vestibular nuclei in off-axis animals overlaps in part with the projection of the otolith afferents. Gacek (1969) and others (Rubertone et al., 1983; Mehler and Rubertone, 1985) have shown that the otolith afferents terminate heavily on the caudal vestibular nuclei (the medial and inferior vestibular nuclei) and the ventral part of the y-group, which receives saccular affercnts. On-axis animals had minimal immunolabeling in these nuclei. In contrast, the $\mathrm{x}$-group of the vestibular complex, which receives ascending projections from the spinal cord (Mehler and Rubertone, 1985), labeled in all animals that were restrained (but greater in the off-axis condition), and there was a distinct lack of labeling in the lateral and superior vestibular nuclei under all conditions. It should be noted that neurons in the lateral vestibular nucleus are sensitive to gravity but there is only a small input directly from the vestibular nerve (Wilson and Jones, 1979).

The inferior olive. The present data suggest that the DMCC, which has been described in many mammalian species (Brodal and Brodal, 1982; Eisenman et al., 1983; Saigal et al., 1983; Whitworth et al., 1983; Rikard et al., 1990), might be one component of the pathways involved in adaptation to a change in the gravito-inertial force. The fastigial nucleus (Brodal, 1976; Courville et al., 1977) and cerebellar uvula (lobule IX; Brodal, 
1980; Sato and Barmack, 1985; Azizi, 1989; Apps, 1990), nodulus (Hoddevik and Brodal, 1977; Whitworth and Haines, 1986), and flocculus (Alley et al., 1975) have been shown to receive climbing fiber inputs from the DMCC. The projection to the uvula and nodulus might be particularly important; lesions of those areas prevent habituation of the time constant of the vestibulo-ocular reflex and the visual and tilt (gravity) suppression of the optokinetic responses (Waespe et al., 1985).

The $\beta$-subnucleus, which receives vestibular as well as visual input (Barmack et al., 1987), also responded to the otolith stimulation, but with more variation among the animals. It should be noted that these olivary neurons were shown by previous investigators to respond only to the direction of passive, horizontal (yaw) rotation of the animal and not to the velocity or frequency of rotation, and it was suggested that these neurons might signal the presence of unintended head movements in a particular direction (Robinson et al., 1988; see also Barmack et al., 1987). In real-life situations, such a movement would give rise to unexpected vestibular and other sensory inputs. The response of the DMCC to a change in the gravito-inertial force sensed by the animal might represent a similar type of response. However, additional experiments would be required to quantify possible differences between the $\beta$-subnucleus and the DMCC.

In summary, the present results show that Fos immunohistochemistry is a reliable and sensitive indicator of brainstem activation in response to prolonged linear acceleration. The data suggest that novel pathways (i.e., vestibulo-olivo-cerebellar, vestibulo-periaqueductal gray, or vestibulo-solitary) might be involved in adaptation to a change in the gravito-incrtial force.

\section{References}

Alley K, Baker R, Simpson JI (1975) Afferents to the vestibulo-cerebellum and the origin of the visual climbing fibers in the rabbit. Brain Res 98:582-589.

Anderson WA, Rutherford JG, Gwyn DC (1983) Midbrain and diencephalic projections to the inferior olive in rat. Proc Can Fed Biol Soc 26:132.

Apps R (1990) Columnar organisation of the inferior olive projection to the posterior lobe of the rat cerebellum. J Comp Neurol 302:236254.

Azizi SA (1989) Principles of organization within the olivocerebellar system in the rat. Exp Brain Res 17:46-51.

Azizi SA, Woodward DJ (1987) Inferior olivary nuclear complex of the rat: morphology and comments on the principles of organization within the olivocerebellar system. J Comp Neurol 263:467-484.

Barmack NH, Mugnaini E, Nelson B (1987) Vestibular modulation of activity of inferior olivary neurons in the beta nucleus of the rabbit. Soc Neurosci Abstr 13:229.

Beitz AJ (1982) The organization of afferent projection to the midbrain periaqueductal gray of the rat. Neuroscience 7:133-159.

Beitz AJ (1985) The midbrain periaqueductal gray in the rat. I. Nuclear volume: cell number, density, orientation, and regional subdivisions. J Comp Neurol 237:445-459.

Beitz AJ (1991) Anatomical and chemical organization of descending pain modulation systems. In: Animal pain (Short CE, Poznak AV, eds), pp 31-62. New York: Churchill Livingston.

Borison HL, Borison R (1986) Motion sickness reflex arc bypasses the area postrema in cats. Exp Neurol 92:723-737.

Brizzee KR, Dunlap WP (1983) Motion-induced alterations in 2-deoxyglucose uptake in brainstem nuclei of squirrel monkeys: autoradiographic and liquid scintillation studies. Brain Behav Evol 23: 14-25.

Brizzee KR, Ordy JM, Mehler WR (1980) Effect of ablation of area postrema on frequency and latency of motion sickness-induced emesis in the squirrel monkey. Physiol Behav 24:849-853.

Brodal A (1976) The olivocerebellar projection in the cat studied with the method of retrograde axonal transport of horseradish peroxidase. 2. The projection to the uvula. J Comp Neurol 166:417-426.
Brodal A (1980) Olivocerebellocortical projection in the cat as determined with the method of retrograde axonal transport of horseradish peroxidase. 2. Topographical pattern in relation to the longitudinal subdivision of the cerebellum. In: The inferior olivary nucleus: anatomy and physiology (Courville J, ed), p 187. New York: Raven.

Brodal P, Brodal A (1982) Further observations on the olivocerebellar projection in the monkey. Exp Brain Res 45:71-83.

Bullitt E (1989) Induction of c-fos-like protein within the lumbar spinal cord and thalamus of the rat following peripheral stimulation. Brain Res 493:391-397.

Bullitt E (1990) Expression of c-fos protein as a marker for neuronal activity following noxious stimulation in the rat. J Comp Neurol 296: 517-530.

Buttner-Ennever JA (1988) Neuroanatomy of the oculomotor system. Amsterdam: Elsevier.

Cass SP, Goshgarian HG (1991) Vestibular compensation after labyrinthectomy and vestibular neurectomy in cats. Otolaryngol Head Neck Surg 104:14-19.

Cass SP, Davidson P, Goshgarian H (1989) Survival of the vestibular nerve after labyrinthectomy in the cat. Otolaryngol Head Neck Surg 101:459-465.

Corcoran ML, Fox RA, Daunton NG (1989) The susceptibility of rhesus monkeys to motion sickness. Av Space Environ Med September:807-809.

Courville J, Augustine JR, Martel P (1977) Projections from the inferior olive to the cerebellar nuclei in the cat demonstrated by retrograde transport of horseradish peroxidase. Brain Res 130:405-419.

Crawford JD, Cadera W, Vilis T (1991) Generation of torsional and vertical eye position signals by the interstitial nucleus of Cajal. Science 252:1551-1553.

D'Amelio FE, Mehler WR, Gibbs MA, Eng LF, Wu J (1987) Immunohistochemical localization of glutamic acid decarboxylase (GAD) and glutamine synthetase (GS) in the area postrema of the cat. Light and electron microscopy. Brain Res 410:232-244.

Dragunow M, Faull R (1989) The use of $c$-fos as a metabolic marker in neuronal pathway tracing. J Neurosci Methods 29:261-265.

Eisenman LM, Sieger DD, Blatt GJ (1983) The olivocerebellar projection to the uvula in the mouse. J Comp Neurol 221:53-59.

Fox RA, Corcoran M, Brizzee KR (1990) Conditioned taste aversion and motion sickness in cats and squirrel monkeys. Can J Physiol Pharmacol 68:269-278.

Fukushima K (1987) The interstitial nucleus of cajal and its role in the control of movements of head and eyes. Prog Neurobiol 29:107192.

Gacek RR (1969) The course and central termination of first order neurons supplying vestibular endorgans in the cat. Acta Otolaryngol [Suppl] (Stockh) 254:1-66.

Gallo M, Arnedo M, Aguero A, Puerto A (1991) Participation of the area postrema in learned aversions induced by body rotation. Behav Brain Res 42:13-23.

Hay M, Bishop VS (1991) Interactions of area postrema and solitary tract in the nucleus-tractus-solitarius. Am J Physiol 260:II1466H1473.

Hirai T, Onodera S, Kawamura K (1982) Cerebellotectal projections studied in cat with horse radish peroxidase or tritiated amino acids axonal transport. Exp Brain Res 48:1-12.

Hoddevik GH, Brodal A (1977) The olivocerebellar projection in the cat studied with the method of retrograde axonal transport of horseradish peroxidase. $V$. The projection to the flocculonodular lobe and the paraflocculus in the rabbit. J Comp Neurol 176:269-280.

Holstege G, Cowie RJ (1990) Nucleus prepositus hypoglossi projects to the dorsolateral periaqueductal gray (PAG): a link between visuomotor and limbic systems. Soc Neurosci Abstr 16:303.3.

Horn KM, DeWitt JR, Nielson HC (1981) Behavioral assessment of sodium arsanilate induced vestibular dysfunction in rats. Physiol Psychol 9:371-378.

Jovanovic-Micic D, Strbac M (1989) Ablation of the area postrema and emesis. Metabol Brain Dis 4:55-60.

Kase M, Nagata R, Kato M (1986) Saccade-related activity of periaqueductal gray matter of the monkey. Invest Ophthalmol Vis Sci 27: $1165-1169$.

Kaufman GD, Anderson JH, Beitz AJ (1991) Activation of a specific vestibulo-olivary pathway following centripetal acceleration in the rat. Brain Res 562:311-317.

Larsen PJ, Moller M, Mikkelsen JD (1991) Efferent projections from 
the periventricular and medial parvicellular subnuclei of the hypothalamic paraventricular nucleus to circumventricular organs of the rat-a Phaseolus-vulgaris-leucoagglutinin (PHA-L) tracing study. J Comp Neurol 306:462-479.

McCrea, RA, Strassman A, Highstein SM (1987) Anatomical and physiological characteristics of vestibular neurons mediating the vertical vestibulo-ocular reflex of the squirrel monkey. J Comp Neurol 264:571-594.

Mehler WR, Rubertone JA (1985) Anatomy of the vestibular nucleus complex. In: The rat nervous system, Vol 2, Hindbrain and spinal cord (Paxinos, G, ed), Chap 9. Marickville, Australia: Academic.

Meller ST, Dennis BJ (1986) Afferent projections to the periaqueductal gray in the rabbit. Neuroscience 19:927-964.

Ostrowska A, Zimny R, Zguczynski L, Sikora E (1990) Subcortical afferents to the interstitial nucleus of Cajal - an anatomical retrograde tracing study in the rabbit. J Hirnforsch 31:747-759.

Paxinos G, Watson C (1986) The rat brain in stereotaxic coordinates, $2 \mathrm{~d}$ ed. Marickville, Australia: Academic.

Rikard BG, Tiork I, Sullivan C, Scheibner T (1990) Distribution of substance P-like immunoreactive fibres and terminals in the medulla oblongata of the human infant. Neuroscience 34:133-148.

Robinson DA (1981) The use of control systems analysis in the neurophysiology of eye movements. Annu Rev Neurosci 4:463-503.

Robinson FR, Fraser MO, Hollerman JR, Tomko DL (1988) Yaw direction neurons in the cat inferior olive. J Neurophysiol 60:17391752.

Rubertone JA, Mehler WR, Cox GE (1983) The intrinsic organization of the vestibular complex: evidence for internuclear connectivity. Brain Res 263:137-141.

Saigal RP, Karamanlidis AN, Voogd J, Michaloudi H, Mangana O (1983) Olivocerebellar connections in sheep studied with the retrograde transport of horseradish peroxidase. J Comp Neurol 217:440448.

Sato Y, Barmack NH (1985) Zonal organization of olivocerebellar projections to the uvula in rabbits. Brain Res 359:281-291.

Schwartz WJ, Sharp FR (1978) Autoradiographic maps of regional brain glucose consumption in resting, awake rats using $\left[{ }^{14} \mathrm{C}\right] 2$-deoxyglucose. J Comp Neurol 177:335-359.

Sharp FR (1976) Rotation induced increases of glucose uptake in rat vestibular nuclei and vestibulocerebellum. Brain Res 110:141-151.

Sudoh M, Shioda K, Kohno M, Ikawa S, Kawakami K, Saiki H (1987) Cardiopulmonary response of rats to centrifugal accelerations. Physiol [Suppl] 30:S135.

Sutton RL, Fox RA, Daunton NG (1988) Role of the area postrema in three putative measures of motion sickness in the rat. Behav Neurol Biol 50:133-152.

Swenson RS, Castro AJ (1983) The afferent connections of the inferior olivary complex in rats. An anterograde study using autoradiographic and axonal degeneration techniques. Neuroscience 8:259-275.

Tran Ba Huy P, Bernard P, Schacht J (1986) Kinetics of gentamicin uptake and release in the rat. J Clin Invest 77:1492-1500.

Waespe W, Cohen B, Raphan T (1985) Dynamic modification of the vestibulo-ocular reflex by the nodulus and uvula. Science 228:199202.

Wang SC, Chinn HI (1954) Experimental motion sickness in dogs. Functional importance of the chemoreceptor trigger zone. Am J Physiol 178:111-116.

Whitworth RH, Haines DE (1986) On the question of nomenclature of homologous subdivisions of the inferior olivary complex. Arch Ital Biol 124:271-317.

Whitworth RH, Haines DE, Patrick GW (1983) The inferior olive of a prosimian primate, Galago senegalensis. II. Olivocerebellar projections to the vestibulocerebellum. J Comp Neurol 219:228-240.

Wilpizeski CR, Lowry LD, Goldman WS (1986) Motion-induced sickness following bilateral ablation of area postrema in squirrel monkeys. Laryngoscope 96:1221-1225.

Wilson VJ, Jones GM (1979) Mammalian vestibular physiology. New York: Plenum.

Zhang SP, Bandler R, Carrive P (1990) Flight and immobility evoked by excitatory amino acid microinjection within distinct parts of the subtentorial midbrain periaqueductal gray of the cat. Brain Res 520: 73-82. 